\title{
Constant-volume vapor-liquid thermal energy storage: Generalized analysis of pure fluids
}

\author{
Abdullah Bamoshmoosh ${ }^{1}$ and Gianluca Valenti ${ }^{1 *}$ \\ *Politecnico di Milano, Dipartimento di Energia, Via R. Lambruschini 4A, 20156, Milano, Italy.
}

\begin{abstract}
Thermal energy storage is of great interest both for the industrial world and for the district heating and cooling sector. Available technologies present drawbacks that reduce the margin of application, such as low energy density, limited temperature range of work, and investment costs. Phase transition is one of the main phenomena that can be exploited for thermal energy storage because of its naturally high energy density. Constant-volume vapor-liquid transition shows higher flexibility and increased heat transfer properties with respect to available technologies. This work presents a description of the behavior of these types of systems. The analysis is carried out through a generalized approach using the Corresponding State Principle. Variation of internal energy as a function of temperature over a fixed range is calculated at constant volume at different values of specific volume. It is shown that, for lower specific volumes, larger temperature ranges of work can be achieved without occurring in the steep pressure increase typically given by the expansion of liquid. Maximum operating temperature range is increased by up to $20 \%$ of the critical temperature with minimal energy loss. In optimal subsets of these ranges of temperature, the energy storage capacity of vapor-liquid systems increases at lower volumes, with energy storage capacity increasing to up to $40 \%$ with a $50 \%$ increase of the reduced volume. This is especially valid for more complex fluids, which are more interesting for these applications because of their higher heat capacity.
\end{abstract}

\section{INTRODUCTION}

The development of energy saving technologies is a key factor for the deployment of sustainable processes. Thermal energy storage systems have been widely investigated because of their role in primary energy saving [1]. The interest for these technologies arises from a number of reasons, such as the need to cover the mismatch between the energy supply and the energy demand or the possibility to exploit a cyclic thermal load that could be present in a process [2].

The main thermal energy storage mechanisms are sensible heat storage, latent heat storage and thermochemical heat storage [3]. Latent thermal energy storage systems are those in which the latent heat released or absorbed by the phase change of a storage medium is exploited to discharge or charge the stored energy in the medium itself. Solid-liquid phase change materials have been developed already, with a wide range of materials being available [4]. However, the applications of these technologies are limited by the low conductivity of these solid-liquid phase change materials, which results in poor heat exchange performances $[5,6]$. On the other hand, vapor-liquid phase change is expected to show much better heat exchange performances with respect to solid-liquid phase change.

Scientific literature shows scarce research over these types of systems. Vapor-liquid phase transition has not been considered for two main reasons. The analysis of Sharma et al. [4] shows that although on mass basis the vapor-liquid latent heat is higher than the solid-liquid latent heat, on volume basis this property is greatly reduced by the much lower density of the vapor phase. In his work, Abhat [7] states that the necessity of pressure-controlled vessels that store a two-phase system rules out the exploitation of this transition because of the increased practical complexity.

This work quantifies the performances of pure fluid vapor-liquid systems in closed and constant volumes from an energy storage point of view. Closed and constant volumes are chosen because they represent best the envisioned application for these energy storage systems. Internal energy of the two-phase volumes as well as their energy storage capacities are evaluated at different reduced densities. Moreover, the variation of internal energy is considered in order to evaluate the change in storage capacity with temperature at different reduced densities.

\footnotetext{
* Corresponding author: gianluca.valenti@polimi.it
} 


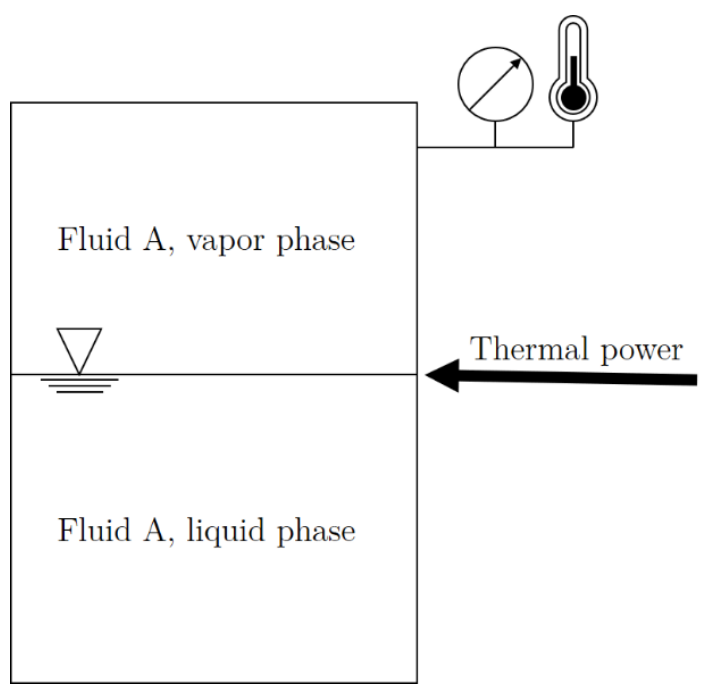

Figure 1. Schematic of the closed volume with pure fluid A and thermal power into the system.
The conceptual approach of this work is to consider the behavior of pure fluids at different specific densities in vaporliquid equilibrium at constant volume. This volume can be schematized as a cylinder filled with a two-phase pure fluid. The free surface level identifies the vapor fraction $\chi$ of the two-phase systems, and thus the overall density $\rho$. A schematic of the system is given in Figure 1.

In this work, thermodynamic properties are usually on volume basis and not mass basis because they represent better the heat storage capacity of closed and constant volumes. This means that whenever internal energy specific to volume is mentioned, it is referred to as $\hat{u}$ (measured for instance in $\mathrm{J} / \mathrm{m}^{3}$ ).

In order to perform a generalized fluid-independent analysis, the three-parameter Corresponding State Principle is used [8]. The principle states that properties of fluids only depend on reduced thermodynamic coordinates and complexity of the fluids themselves. This allows evaluation of the energy stored by different types of fluids in two-phase vapor-liquid systems over a fixed reduced temperature range at the same overall reduced he results obtained in this work for most fluids.

\section{FLUID MODEL}

This section gives an overview of the formulation of the Corresponding State Principle that is used in this work to calculate the fluid properties. The formulation of the principle is the Lee-Kesler one [9, 10], in which the acentric factor is used as a measure of the molecular complexity of the fluid. This approach consists in the calculation of the properties of a simple and a reference complex fluid at the requested reduced temperature and pressure. The difference between reference complex fluid property and the simple fluid property is divided by the acentric factor of the reference fluid $\omega^{r}$, which in the Lee-Kesler formulation is n-octane, and then multiplied by the acentric factor of the actual fluid of interest $\omega$. The scaled difference is added to the calculated simple reference fluid property. Mixing rules for critical properties are not needed because focus is on pure fluids. Mathematically, the compressibility factor $Z$ is

$$
Z=\frac{P v}{R_{g} T}=Z_{0}\left(T_{r}, P_{r}\right)+\frac{\omega}{\omega_{r}} Z_{l}\left(T_{r}, P_{r}\right)
$$

where $Z_{0}$ and $Z_{1}$ are two different functions in terms of reduced temperature and pressure. These two functions represent the compressibility factor of a simple fluid, $Z_{0}$, and the difference between compressibility factor of the reference complex fluid and the simple fluid, $Z_{l}$. The Lee-Kesler Equation of State needs to be expressed in temperature and volume to be able to describe the stated problem. The $Z_{0}$ and $Z_{1}$ functions are not per se functions of the actual specific volume $v$, which is only included in the mathematical expression through the compressibility factor definition. This means that, even though the function has the same mathematical form of a Benedict-Webb-Rubin-Starling Equation of State and could be solved with similar algorithms [11, 12], it cannot be solved directly for volume, because it is not explicit in it. It rather needs a double numerical method, one for the solution of each function $Z_{0}$ and $Z_{1}$ in temperature and pressure and one for the solution of the overall system in temperature and volume.

The factors of the solution $Z$, i.e. $Z_{0}$ and $Z_{1}$, are used to calculate the residual properties of the fluids, i.e. the difference between the value of the property $\pi$ and the value of the same property in a fictitious ideal gas state $\pi^{i g}$. In the Lee-Kesler approach, any thermodynamic residual $\pi^{r e s}$ can be written with the same formulation as for $Z$ :

$$
\pi^{r e s}=\pi_{0}\left(T_{r}, P_{r}, Z_{0}\right)+\frac{\omega}{\omega_{r}} \pi_{1}\left(T_{r}, P_{r}, Z_{1}\right)
$$

In this case, internal energy residual on mass basis is calculated from the enthalpy residual, which is a relatively simple function of $Z_{0}$ and $Z_{1}$. From the definitions of residual property and enthalpy it can be found that

$$
\begin{gathered}
\Delta h=\Delta u+p v \\
\Delta h^{i g}=\Delta u^{i g}+p v^{i g}=\Delta u^{i g}+R_{g} T \\
\Delta h=\Delta h^{i g}+\Delta h^{r e s}=\Delta u^{i g}+\Delta u^{r e s}+p v=\Delta h^{i g}-R_{g} T+\Delta u^{r e s}+p v \\
\Delta u^{r e s}=\Delta h^{r e s}-p v+R_{g} T
\end{gathered}
$$

Vapor-liquid equilibrium needs to be accounted for in the calculation of phase properties for two-phase systems. For pure fluids saturation pressure is easily calculated. Since each function $Z_{0}$ and $Z_{1}$ represent the volumetric behavior of reference fluids in reduced temperature and pressure, they show a sharp variation around specific values of the reduced pressure. This 
information combined leads to a simple formulation of the saturation pressure, which is provided by Lee and Kesler themselves [9]. Once the saturation pressure is calculated, saturated liquid and saturated vapor compressibility factors can be obtained with a simpler temperature-pressure formulation of the Equation of State. For pure fluids, this simplifies the calculation of the vapor fraction $\chi$ greatly. This is because the dew and bubble pressures are equal, and the two phases have same unity composition in the saturated phases.

To calculate the properties of the overall vapor-liquid system, calculation of residual properties needs to be done twice, once for the $Z_{0 v}$ and $Z_{l v}$ pair to calculate the saturated vapor compressibility factor $Z_{v}$, and once for the $Z_{0 l}$ and $Z_{l l}$ pair to calculate the saturated liquid compressibility factor $Z_{l}$. Once the vapor fraction and the phase residual properties are calculated, overall properties can be obtained through the leverage rule. The internal energy residual function can be thus evaluated at various temperatures and fixed specific volume.

The Lee-Kesler Equation of State does not present a way to calculate the ideal gas properties, but only provides the residual properties. The calculation of the ideal gas internal energy is a widely studied topic [13], and is usually treated with polynomial approximations that are fluid-specific. In order to maintain the general approach of the Lee-Kesler Equation of State, the assumption of constant specific heat is done. This leads to an important simplification while keeping a generalized approach, accounting for different behavior of different fluids in the ideal gas state.

\section{GENERALIZED STUDY}

This section shows the adopted procedure for generalizing the proposed analysis and the considered thermodynamic conditions. According to the generalized Lee-Kesler Equation of State, fluid properties are only dependent on the reduced coordinates of its state and its molecular complexity. Classes of fluids can be defined based on their molecular complexity to study the change of behavior of these fluids. Different values of the acentric factor $\omega$ can be assigned to different hypothetical fluids, representing the various complexity classes they could have. Given that n-octane is used as a reference complex fluid, with $\omega^{r}$ equal to 0.398 , an acentric factor of 0.4 is chosen to refer to complex fluids, 0 to simple fluids, and 0.2 to fluids with intermediate behavior. Some reference fluids are used to test the validity of this approach, as listed in Table 1 . These fluids are chosen to cover as uniformly as possible the reasonable $\omega$ spectrum.

Fluid complexity has a major impact on the ideal gas behavior of the fluids. The same classes of fluids are used to define the ideal gas specific heat $c_{v}^{i g}$ for continuity of results. The classes of fluids defined in Table 1 overlap the classical monoatomic-linear-polyatomic molecule classification that is used to account for the effect of molecular degrees of freedom $c_{v}^{i g}$ with a good degree of accuracy. The choices of the values for the constant ideal gas specific heat are $c_{v}^{i g}=3 / 2 R_{g}$ for simple fluids, $c_{v}^{i g}=5 / 2 R_{g}$ for intermediate fluids and $c_{v}^{i g}=3 R_{g}$ for complex fluids.

Pure fluids are tested in the range of $v_{r}$ between 0.3 and 500 as well as of $T_{r}$ between 0.4 and 0.95 . This temperature range allows coverage of a significant portion between the triple point temperature and the critical temperature for all fluids. Going below the triple point temperature or above the critical temperature would be meaningless for this work because vapor-liquid equilibrium does not occur outside this window. The volume range is chosen in order to allow studying the effect of vapor liquid equilibrium in the whole $T_{r}$ spectrum. The value of 0.3 for reduced volume is considered to have a comparison with a one-phase liquid fluid, which will not undergo phase change.

Table 1. Acentric factors of sample fluids and the complexity classes they fall into.

\begin{tabular}{ccc}
\hline Class & Fluid & $\boldsymbol{\omega}$ \\
\hline Simple & Argon & 0.001 \\
& Nitrogen & 0.040 \\
\cline { 2 - 3 } Intermediate & Chlorine & 0.074 \\
& Nitrous Oxide & 0.143 \\
\cline { 2 - 3 } & Carbon Dioxide & 0.239 \\
\hline \multirow{2}{*}{ Complex } & Water & 0.344 \\
\hline
\end{tabular}




\section{RESULTS}

This section provides the overall results obtained by this analysis. An analysis of the countercondensation phenomenon is given. An energy storage analysis is then given, first in the extended volume range of interest and then in the more specific area of overall reduced volume lower than the critical volume.

\section{Phenomenon description and countercondensation effect}

Constant-volume processes are represented well in the volume-temperature diagram, as in Figure 2. On this diagram there are two distinct areas: one at an overall specific volume lower than the critical one $\left(v<v_{c}\right)$, on the left, and one at higher $\left(v>v_{c}\right)$, on the right. These two regions show different phase change behavior. Figure 2 depicts the processes at reduced volumes of 0.45 and 45 . The first crosses the saturated liquid while the second the saturated vapor line.

Figure 3 shows the behavior of vapor fraction as a function of temperature for the two cases. In volume-constant processes higher than critical, increasing the temperature leads to an increase in the vapor fraction and, eventually, to a complete vapor phase. At lower than critical, increasing the temperature leads to an increase of the vapor fraction at first, but close to saturation to a decrease in vapor fraction. The phenomenon for which an increase in temperature eventually leads to a decrease of the vapor fraction will be called countercondensation in this work. It is the reason for which when saturation temperature is eventually reached in this process, the system behaves as pressurized liquid tanks at temperatures above their maximum operating temperature. While on the right side of the diagram pressure in the two phase grows exponentially with temperature and then grows more or less linearly after saturation, on the left side of the diagram it keeps growing steeply. After the saturation curve is crossed, systems that go through countercondensation experience an increase in pressure that is up to two orders of magnitude than the ones that do not. This is because for liquids, the higher the temperature, the stronger the pressure energy needs to be in order to contain the thermal expansion forces and keep specific volume constant [14].

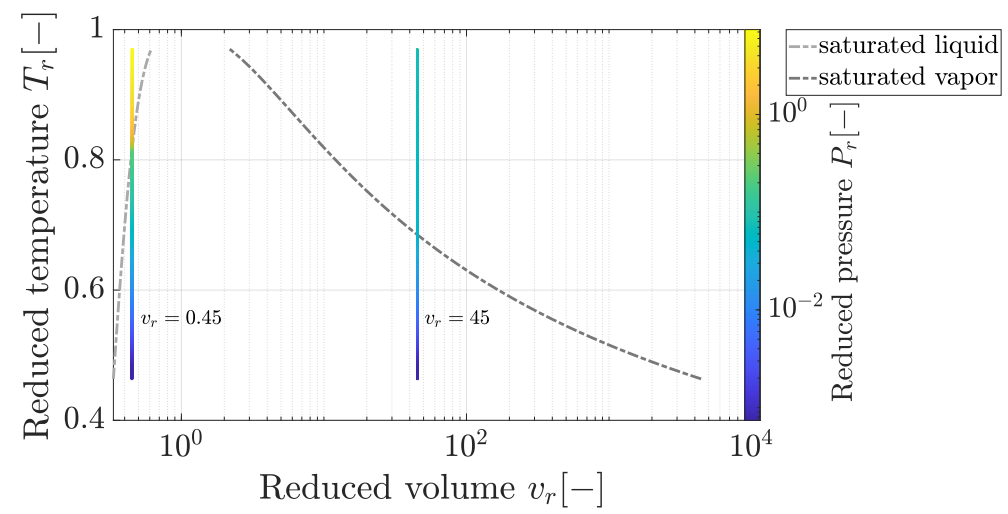

Figure 2. Reduced temperature $T_{r}$, as a function of reduced volume $v_{r}$, at reduced volume of 0.45 and 45 , taken as example, for a hypothetical intermediate complexity fluid with acentric factor $\omega$ equal to 0.2 , with colorbar for reduced pressure $P_{r}$.

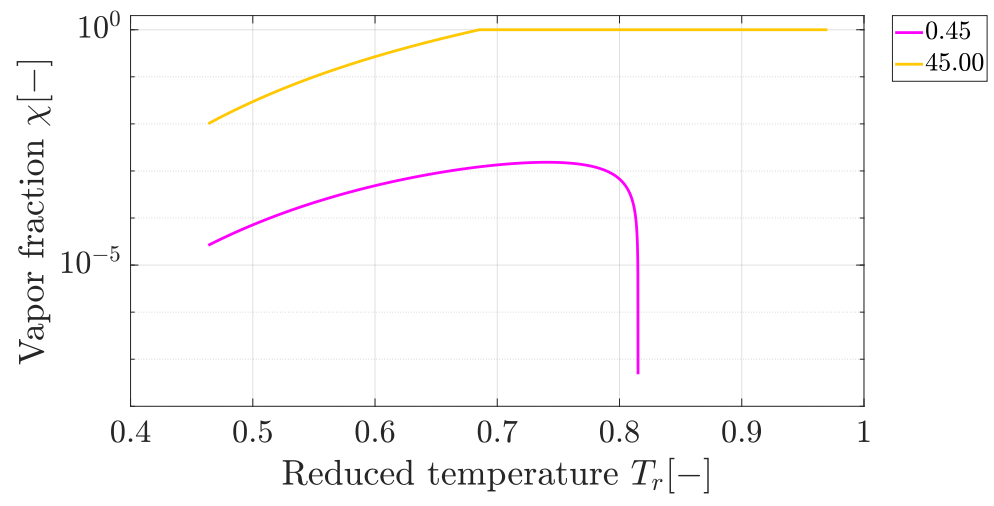

Figure 3. Vapor fraction $\chi$ as a function of reduced temperature $T_{r}$, at reduced volume of 0.45 and 45 , taken as example, for hypothetical with acentric factor $\omega$ equal to 0.2 . 


\section{Extended volume range analysis}

This section focuses on the whole reduced volume range, from 0.3 to 500 , for an intermediate complexity fluid by three diagrams. The $v_{r}-T_{r}$ diagram shows the different density levels of the analysis, defining the target range of temperature and reduced volume. The $T_{r}-\Delta \hat{u}_{r}$ diagram shows the thermal energy storage capacity on a volume basis, indicating how much thermal energy can be stored over the temperature range. Lastly, the $T_{r}$ - $\partial \hat{u}_{r} / \partial T_{r}$ shows the local heat capacity at a specific temperature, indicating which system stores better the heat at a given temperature.

Figure 5 shows the various processes on the first type of diagrams, with six reduced density levels. Figure 6 shows how much energy is stored for each of those processes at each of those different levels of reduced volumes. Increasing the specific volume does not improve heat storage capacity. A sharp decrease can be seen between the first two curves at $v_{r}$ equal to 0.3 and 1 because, although the mass specific storage capacity does not vary dramatically (it even increases, as can be seen in Figure 6), density variation has a predominant effect on the heat storage capacity.

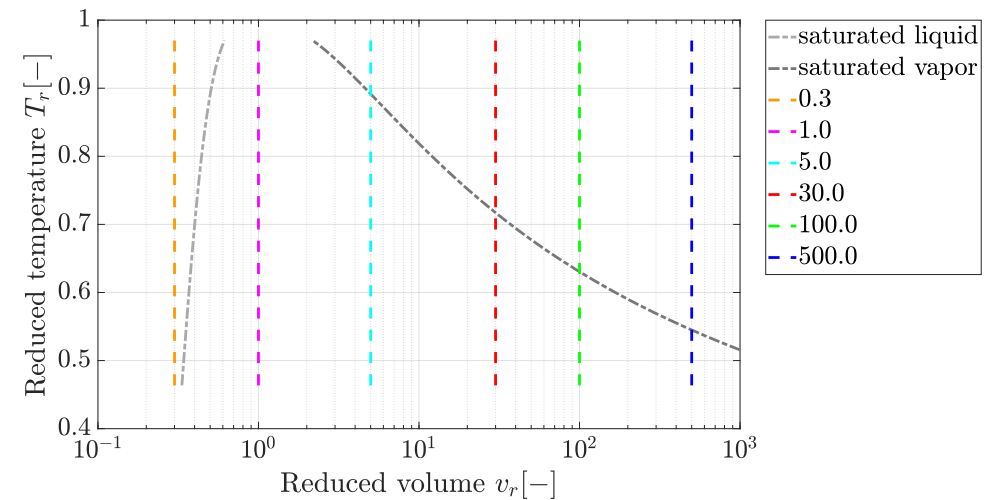

Figure 4. Reduced temperature $T_{r}$ as a function of reduced volume $v_{r}$ for a hypothetical fluid with $\omega$ equal to 0.2 .

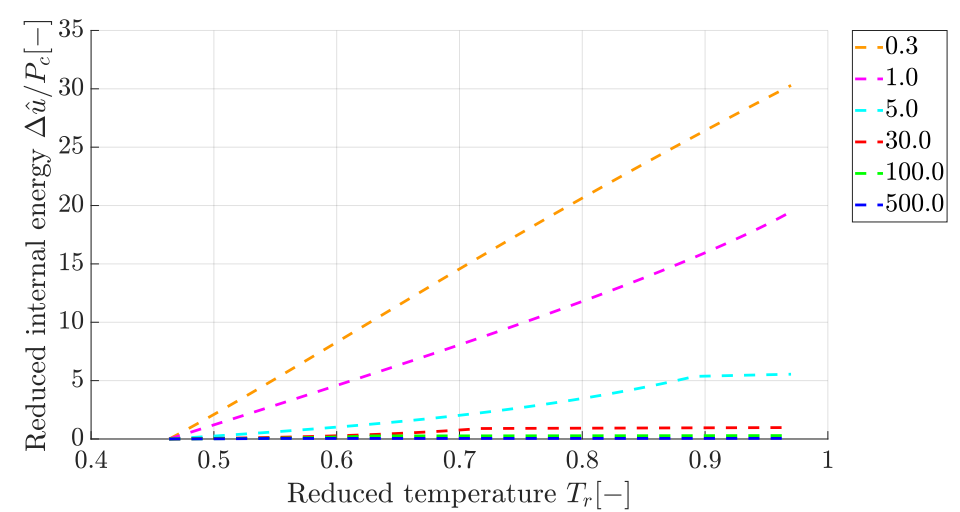

Figure 5. Reduced internal energy on volume basis $\Delta \hat{u}_{r}$ as a function of $T_{r}$ (for a hypothetical fluid with $\omega$ equal to 0.2 .

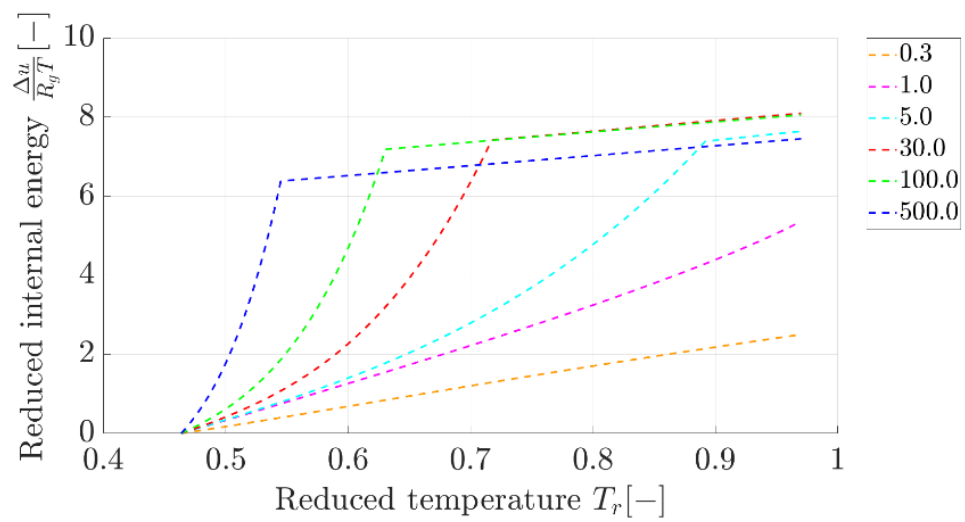

Figure 6. Reduced internal energy on mass basis $\Delta u_{r}$ as a function of reduced temperature $T_{r}$ for a fluid with $\omega$ equal to 0.2 . 


\section{Analysis at overall volume below critical}

The results from the extended volume range indicate that systems at the right of the critical volume $v_{c}$, are not of interest. This result is not limited to the case of intermediate complexity fluids, but can be extended to any fluid. Hence, this section focuses on the values of reduced volume only between 0.30 and 0.90 . Approaching closely the critical volume $v_{c}$ would be useless because the conditions of the fluid would get too close to the critical point and the margin for exploitation of the phase change would be limited, since the two phases get more and more similar.

Figure 7 shows the $v_{r}-T_{r}$ diagram for all tests run in this range. The diagrams are fairly similar to each other, since the reduced coordinates are the same. A change in the slope of the saturated liquid curve can be seen, where for more complex fluids the saturation temperature goes higher. This leads to a change in the point of countercondensation, in particular moving it at higher reduced temperatures for more complex fluids.
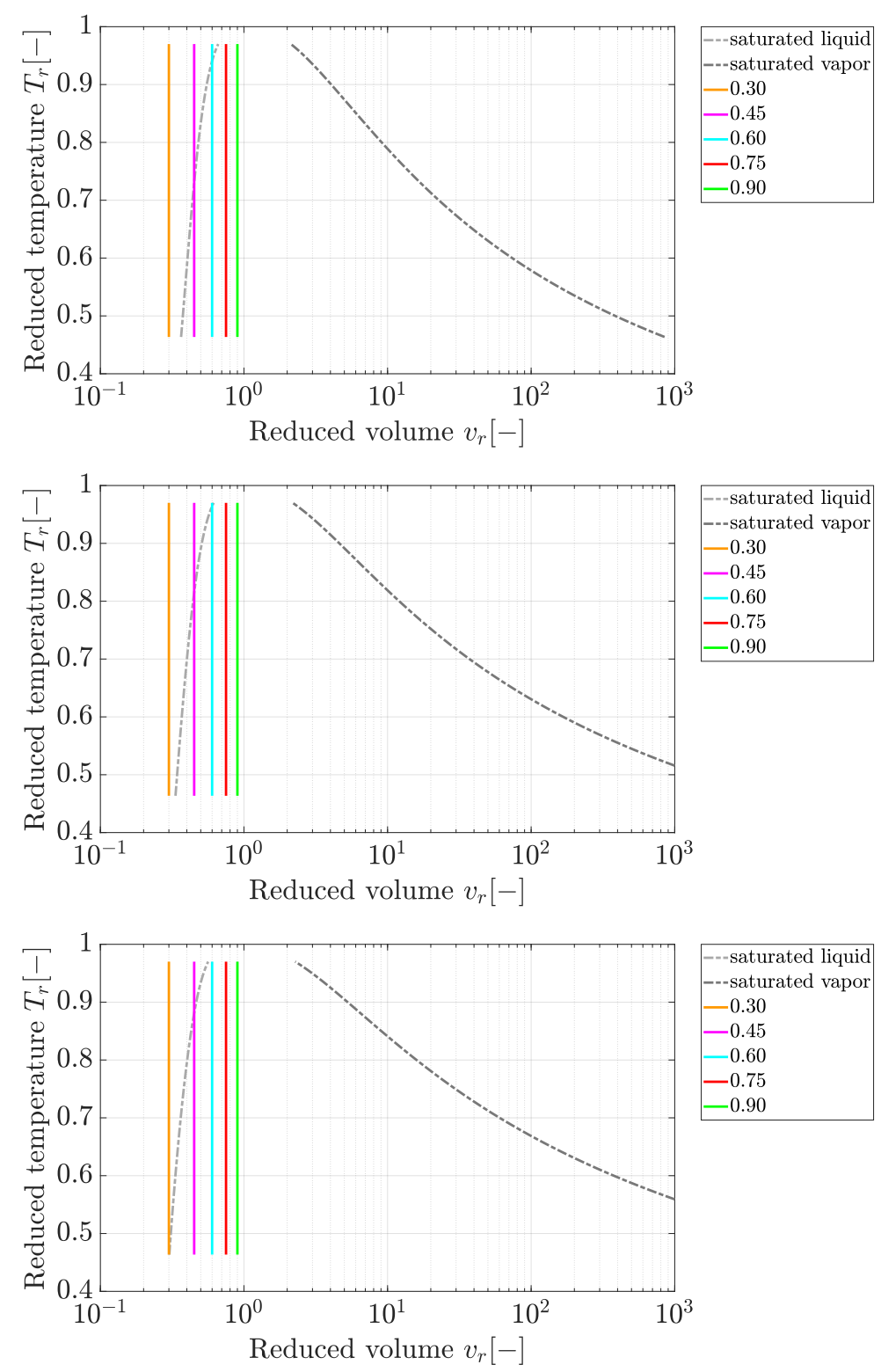

Figure 7. Reduced temperature $T_{r}$ as a function of reduced volume $v_{r}$ for hypothetical fluid with $\omega$ equal to 0 (top), equal to 0.2 (middle) and equal to 0.4 (bottom) with reduced volume from 0.3 to 0.9 . 
Figure 8 shows the internal energy storage capacity on volume basis as a function of temperature for the three hypothetical fluids. A discontinuity corresponding to countercondensation can be seen for all curves that cross the saturation curve in Figure 7, e.g. the curve with reduced volume equal to 0.45 for simple fluids has a discontinuity at a temperature that is at $20 \%$ of the critical temperature lower than the one at reduced volume equal to 0.60 . This discontinuity leads to a sharp change in the slope of the curve, which means less energy stored per unit temperature increment. Figure 8 also shows how the curves at higher reduced volume start showing higher energy storage capacity with respect to the purely liquid condition, which is the one at reduced volume equal to 0.30 . This is despite having up to $50 \%$ higher specific volume, as in the case of complex fluids, where the curve at reduced volume equal to 0.45 shows up to $70 \%$ more energy stored as the curve at reduced volume equal to 0.30 , which is the pure liquid.
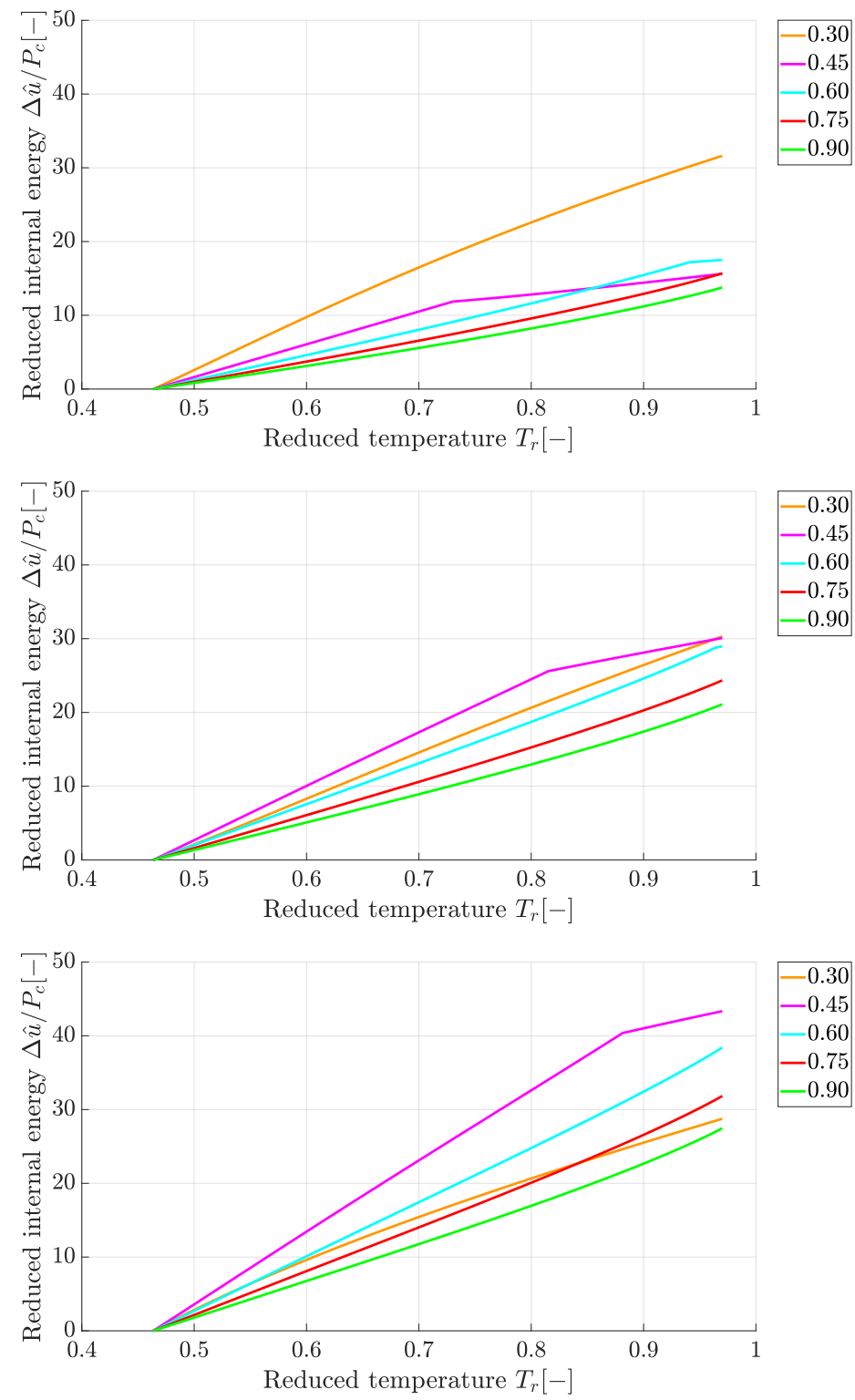

Figure 8. Reduced internal energy on volume basis $\Delta \hat{u}_{r}$ as a function of reduced temperature $T_{r}$ for hypothetical fluid with $\omega$ equal to 0 (top), equal to 0.2 (middle) and equal to 0.4 (bottom) with reduced volume $v_{r}$ from 0.3 to 0.9 . 
Figure 9 shows the local variation of internal energy as a function of reduced temperature, with additional information on the pressure of the system itself through a logarithmic colormap. The pressure change after countercondensation moves to higher temperatures for more complex fluids. Internal energy shows the same pattern as in Figure 8, with low reduced volume systems having up to twice the capacity than the pure liquid systems at high complexity fluids for specific reduced temperatures. Lower volume systems show lower pressures at same temperature, which means that some energy storage capacity could be sacrificed to have wider operating ranges of temperature regardless of the complexity of the fluid itself.
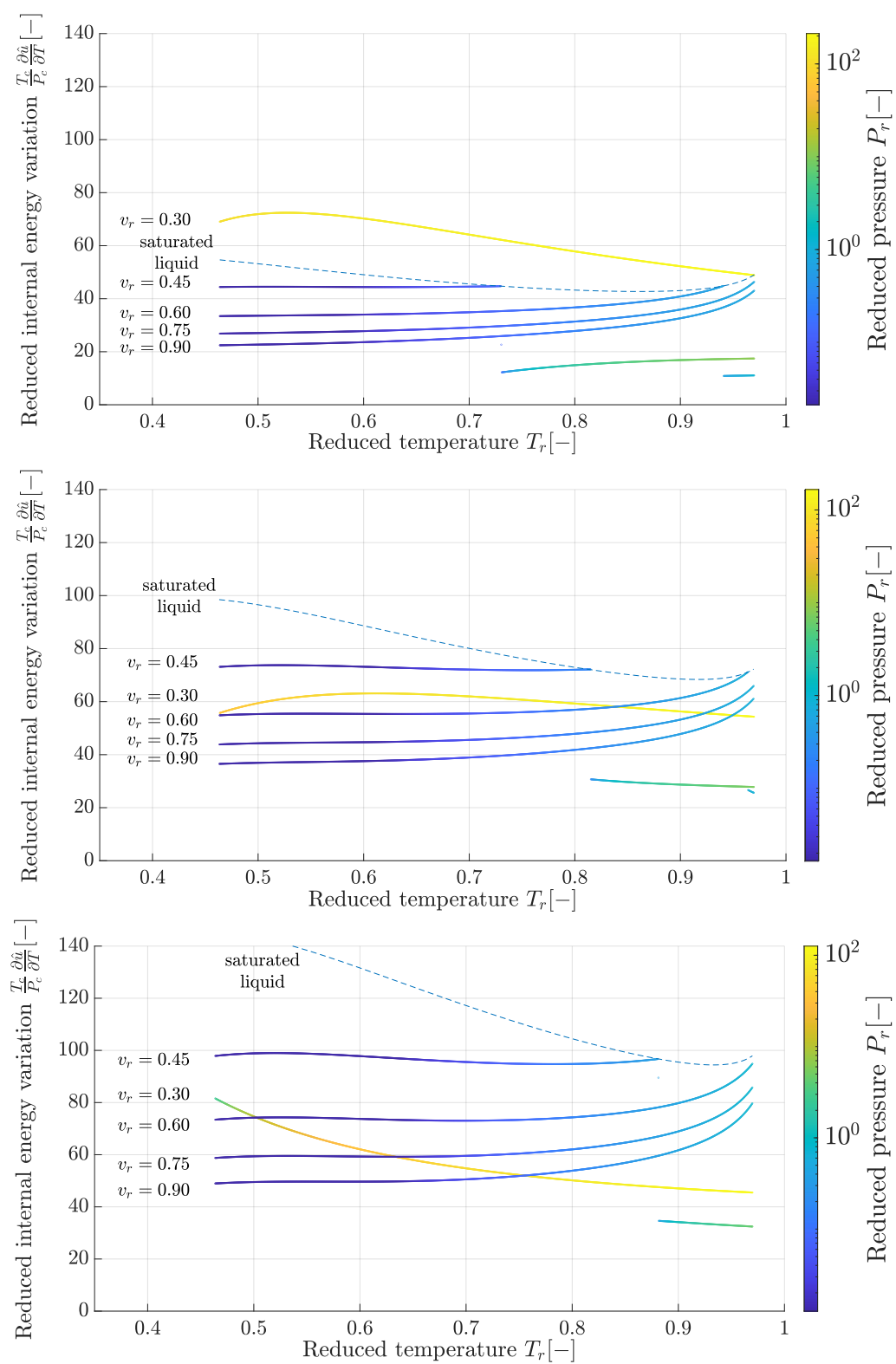

Figure 9. Variation of reduced internal energy on volume basis $\partial \hat{u}_{r} / \partial T_{r}$ as a function of reduced temperature $T_{r}$ for hypothetical fluids, with acentric factor $\omega$ equal to 0 (top), equal to 0.2 (middle) and equal to 0.4 (bottom) with reduced volume $v_{r}$ from 0.3 to 0.9 and with colorbar for reduced pressure $P_{r}$. 


\section{DISCUSSION}

Energy storage sharply decreases at volumes higher than critical volume, as shown by Figure 6 . Systems at reduced volume $v_{r}$ equal to 0.3 store almost twice as much energy than systems at $v_{r}$ equal to 1 . In the new range of interest, i.e. at volumes lower than critical, complexity of the systems affects their saturation curve, and thus countercondensation. For example, countercondensation temperature for the curve at $v_{r}$ equal to 0.6 , as illustrated by Figure 7, is higher for intermediate systems than for simple ones; in their turn complex systems do not even reach countercondensation at all in the range of interest, leading to larger temperature ranges that can be exploited at increasing $\omega$ (Figure 8). Furthermore, the energy variation with temperature increases with complexity (Figure 9). Complex fluids show internal energy variation for two-phase systems that are around twice as much as the ones of simple fluids. For single-phase systems, i.e. curves at $v_{r}$ equal to 0.3 , this property seems comparable regardless of complexity. Single-phase systems are shown to not be relevant for this analysis since they present very high pressures that are consistent with volume-constant processes far above the countercondensation temperature.

\section{CONCLUSIONS}

The analysis done in this work considers two-phase constant volume processes through a generalized approach. An analysis of high and low reduced volume processes is carried out. High focus is then put on lower specific reduced volume. Results of this analysis shows mainly that:

- The increase of pressure of constant volume processes after countercondensation leads to an increase in pressure of up to two orders of magnitude with respect to processes at volume higher than critical;

- Molecular complexity of the fluids is the main parameter for the energy storage. Simpler fluids do not show an improvement in the energy storage at higher reduced volumes, and they also show overall lower energy storage capacity on volume basis in all cases;

- Thermal energy storage could be enhanced by exploiting vapor-liquid equilibrium with respect to sensible liquid thermal energy storage for fluids of higher complexity. Results show storage up to almost twice the energy storage of one-phase system with fluids of $50 \%$ higher overall specific volume;

- Two-phase systems have a wider range of operating temperatures where it is possible to think of an application of these systems. Increasing specific volume allows exploiting the system for ranges of temperature that can be up to $20 \%$ of the critical temperature $T_{c}$ wider, with pressures being up to two orders of magnitude lower at the same temperature;

The continuation of this work needs to show:

- A description of the behavior of multicomponent systems. Major interest needs to be put on two-phase mixtures because it could allow tuning the design parameters of those energy storage systems at need;

- A deepened analysis of third-body interactions that could improve vapor-liquid equilibrium from an energy storage point of view;

- An application of these processes in an existing plant or processes.

NOMENCLATURE

$c_{v} \quad$ Specific heat

$h \quad$ Specific enthalpy on mass basis

$\hat{h} \quad$ Specific enthalpy on volume basis

$P$ Pressure

$R_{g} \quad$ Specific gas constant

$R_{u} \quad$ Universal gas constant

$T$ Temperature

$u \quad$ Specific internal energy on mass basis

$\hat{u} \quad$ Specific internal energy on volume basis

$v$ Specific volume

$Z \quad$ Compressibility factor

$\pi \quad$ Generic property

$\rho$ Density

$\begin{array}{cl}\chi & \text { Vapor fraction } \\ \omega & \text { Acentric factor } \\ \text { Superscripts } & \\ i g & \text { Ideal gas } \\ r & \text { Reference } \\ r e s & \text { Residual } \\ \text { Subscripts } & \\ 0 & \text { Simple } \\ l & \text { Difference between complex and simple } \\ c & \text { Critical } \\ l & \text { Liquid } \\ r & \text { Reduced } \\ v & \text { Vapor }\end{array}$




\section{REFERENCES}

[1] P. Arce, M. Medrano, A. Gil, E. Oró and L. F. Cabeza, "Overview of thermal energy storage (TES) potential energy savings and climate change mitigation in Spain and Europe," Applied Energy, vol. 88, pp. 2764-2774, 2011.

[2] S. Kalaiselvam and R. Parameshwaran, Thermal Energy Storage Technologies for Sustainability, Academic Press, 2014.

[3] L. F. Cabeza, I. Martorell, L. Mirò, A. Fernàndez and C. Barrenche, "Introduction to thermal energy storage," in Advances in Thermal Energy Storage Systems, Methods and Applications, Woodhead Publishing, 2015.

[4] A. Sharma, V. V. Tyagi, C. R. Chen and D. Buddhi, "Review on thermal energy storage with phase change materials and applications," Renewable and Sustainable Energy Reviews, vol. 13, no. 2, pp. 318-347, 2009.

[5] Z. A. Qureshi, H. M. Ali and S. Khushnood, "Recent advances on thermal conductivity enhancement of phase change materials for energy storage systems: A review," International Journal of Heat and Mass Transfer, vol. 127, pp. 838856, 2018.

[6] S. Wu, T. Yan, Z. Kuai and W. Pan, "Thermal conductivity enhancement on phase change materials for thermal energy storage: A review," Energy Storage Material, vol. 25, pp. 251-295, 2020.

[7] A. Abhat, "Low Temperature Latent Heat Thermal Energy Storage: Heat Storage Materials," Solar Energy, vol. 30, no. 4, pp. 313-332, 1983.

[8] R. Rota, Fondamenti di Termodinamica dell'Ingegneria Chimica, Pitagora, 2015.

[9] B. I. Lee and M. G. Kesler, "A Generalized Thermodynamic Correlation Based on Three-Parameter Corresponding States," AIChE Journal, vol. 21, no. 3, pp. 510-527, 1975.

[10] U. Plöcker, H. Knapp and J. Prausnitz, "Calculation of High-Pressure Vapor-Liquid Equilibria from a CorrespondingStates Correlation with Emphasis on Asymmetric Mixtures," Industrial \& Engineering Chemistry Process, Design and Development, vol. 17, no. 3, pp. 324-322, 1978.

[11] K. E. Starling, "Thermo Data Refined for LPG, Part 1: Equation of State and Computer Prediction," Hydrocarbon Processing, vol. 50, no. 3, pp. 101-104, 1971.

[12] M. B. Mills, J. M. Wills and V. L. Bhirud, "The Calculation of Density by the BWRS Equation of State In Process Simulation Contexts," AIChE Journal, vol. 26, no. 6, 1980.

[13] B. E. Poling, J. E. Prausnitz and J. P. O'Connell, The Properties of Gases and Liquids, McGraw-Hill, 2001.

[14] M. Taravillo, V. G. Baonza, M. Cáceres and J. Núñez, "Thermodynamic regularities in compressed liqudis: I. The thermal expansion coefficient," Journal of Physics: Condensed Matter, vol. 15, no. 19, pp. 2979-2989, 2003.

[15] E. Macchi and A. Perdichizzi, "Efficiency Prediction for Axial-Flow Turbines Operating With Nonconventional Fluids," Journal of Engineering for Power, vol. 103, no. 4, pp. 718-724, 1981. 